\title{
Leidenfrost Rotor Dynamics: Design of Turbine-inspired Substrates
}

\author{
Prashant Agrawal' ${ }^{1}$, Gary G. Wells ${ }^{1}$, Rodrigo Ledesma-Aguilar ${ }^{1}$, Glen McHale ${ }^{1}$, Anthony Buchoux ${ }^{2}$, \\ Adam Stokes', Anthony Walton², Jonathan Terry ${ }^{2}$, Khellil Sefiane ${ }^{2}$ \\ ${ }^{1}$ Smart Materials \& Surfaces Laboratory, Northumbria University, \\ E-block, Ellison Building, Newcastle upon Tyne, NE1 8ST, UK \\ prashant.agrawal@northumbria.ac.uk; gary.wells@ northumbria.ac.uk; rodrigo.ledesma@ northumbria.ac.uk; \\ glen.mchale@northumbria.ac.uk \\ ${ }^{2}$ School of Engineering, University of Edinburgh, \\ The King's Building, Edinburgh, EH9 3JL, UK \\ anthony.buchoux@ed.ac.uk; adam.stokes@ed.ac.uk; anthony.walton@ed.ac.uk; jon.terry@ed.ac.uk; k.sefiane@ed.ac.uk
}

\section{Extended Abstract}

The Leidenfrost effect is a case of thin-film boiling where a liquid droplet levitates over a surface heated to temperatures significantly higher than the liquid's boiling point [1]. The vapour layer on which the droplet levitates significantly reduces friction and increases the droplet's lifetime due to reduced thermal conductivity. These highly mobile droplets can also selfpropel by asymmetrically texturing the substrate [2]. The asymmetry rectifies the vapour flow, which produces a viscous drag on the levitating component and results in a directed propulsion. We have previously shown that this vapour rectification can be combined with a turbine-like substrate to create a new conceptual heat engine based on rotating liquids and (sublimating) solids [3]. The liquid (or the solid) acts as a working substance, converting heat from the substrate into work in the form of rotation. We have also recently shown that by continuously replenishing the liquid, we can sustain this energy conversion and transfer the rotation to additional non-volatile solid components, demonstrating a closed thermodynamic cycle operation of this Leidenfrost engine [4].

The work output in this Leidenfrost heat engine is dictated by viscosity dominated shear forces as opposed to conventional steam turbines which rely on momentum transfer to the rotor [5]. In this work, we experimentally and numerically investigate the rotation of a liquid volume, coupled to a solid plate, levitating over a heated texture substrate; this coupled solid-liquid system is termed as a Leidenfrost rotor. We employ 3D numerical simulations to design substrates, which maximize torque from the viscous forces on the liquid-vapour interface. We then experimentally assess the rotation characteristics of these designs by observing the rotation of the supported solid plate over time. We find that substrate geometries based on a logarithmic curve design produce the maximum torque. As the substrate sizes used are of the order of $10 \mathrm{~mm}$, vapour bubbles form in the liquid volume due to the high vapor pressure [6]. The bubble formation reduces liquid coverage, which decreases torque, and affects the rotation stability of the plate. Through analytical models we predict this bubble formation and find that substrates with high groove depth reduce bubble formation and increase the power output and rotation stability. The model shows good agreement with experiments. By investigating the operating principles of these Leidenfrost rotors, we enable the development of novel heat engines employing levitation.

Due to the inherent extremely low-friction operation, these Leidenfrost heat engines promise new avenues for power generation at macro and micro scales. At microscales, the energy cost of levitation is low and the virtually frictionless vapour bearing significantly reduces frictional losses due to a high surface area to volume ratio. At macroscales, these engines have potential applications in low-gravity environments such as space and planetary exploration. Such engines can utilize the heat cycles of naturally occurring alternative working substances [7], such as liquids and ices of $\mathrm{H}_{2} \mathrm{O}, \mathrm{CO}_{2}$ and $\mathrm{CH}_{4}$, in extreme conditions of high temperature differences and low pressures for thermal energy harvesting.

\section{Acknowledgement}

We would like to thank EPSRC for funding (EP/P005896/1 and EP/P005705/1). 


\section{References}

[1] J. G. Leidenfrost, "On the fixation of water in diverse fire," Int. J. Heat Mass Transf., vol. 9, pp. 1153-1166, 1966 (Translated by Carolyn Wares).

[2] G. Lagubeau, M. Le-Merrer, C. Clanet and D. Quéré, "Leidenfrost on a ratchet," Nat. Phys., vol. 7, no. 5, pp. 395-398, 2011.

[3] G. G. Wells, R. Ledesma-Aguilar, G. McHale and K. A. Sefiane, “A Sublimation Heat Engine,” Nat. Commun., vol. 6, pp. 6390, 2015.

[4] P. Agrawal, G. G. Wells, R. Ledesma-Aguilar, G. McHale, A. Buchoux, A. Stokes and K. A. Sefiane, "Leidenfrost heat engine: Sustained rotation of levitating rotors on turbine-inspired substrates," Applied Energy, 2019.

[5] P. K. Nag, Power Plant Engineering, Tata McGraw-Hill Education, New Delhi, 2002.

[6] F. Celestini, T. Frisch, A. Cohen, C. Raufaste, L. Duchemin, and Y. Pomeau, "Two dimensional leidenfrost droplets in a Hele-Shaw cell," Phys. Fluids, vol. 26, no. 3, 2014.

[7] L. Sibille, J. G. Mantovani and J. A. Dominguz, "In-Space Propulsion Engine Architecture Based on Sublimation of Planetary Resources: From Exploration Robots to NEO Mitigation,” Earth and Space, pp. 479-488, 2012. 